\title{
INTRODUCCIÓN: «DIÁLOGOS ENTRE LA DEMOCRACIA PARTICIPATIVA Y LA INTERSECCIONALIDAD. CONSTRUYENDO MARCOS PARA LA JUSTICIA SOCIAL»
}

\author{
Patricia MARTÍNEZ-GARCÍA \\ patmartinez.garcía@gmail.com \\ Aradia, espacio para la profundización democrática, S. Coop. Pequeña \\ Departamento de Ciencia Política y de la Administración \\ Facultad de Ciencias Sociales y de la Comunicación (UPV/EHU) \\ Grupo de investigación Parte Hartuz: estudio sobre la democracia participativa \\ Jone MARTínEZ-PALACiOS \\ jone.martinez@ehu.eus \\ Departamento de Ciencia Política y de la Administración \\ Facultad de Ciencias Sociales y de la Comunicación (UPV/EHU) \\ Grupo de investigación Parte Hartuz: estudio sobre la democracia participativa
}

En los últimos años, la democracia participativa y la interseccionalidad se han convertido en dos ámbitos referentes para la consecución de una mayor justicia social. Sin embargo, esta búsqueda ha discurrido de forma paralela entre ambas herramientas, lo que ha limitado la exploración de la heurística que puede surgir de su encuentro. Así, este número 33 de la revista Feminismo/s busca contribuir al diálogo entre ambas perspectivas, experimentando con las posibilidades que ofrece esta relación para observar, analizar y transformar nuestros entornos.

El interés en esta aventura no es nuevo, sino que procede de un trabajo iniciado en el eje Democracia y Feminismos del grupo de investigación Parte Hartuz de la Universidad del País Vasco/Euskal Herriko Unibertsitatea y que

Los contenidos de la revista se publican bajo una licencia de Creative Commons Reconocimiento 4.0 Internacional (CC BY 4.0)

Feminismo/s 33, junio 2019, pp. 13-20 
se ha ido concretando en diferentes proyectos. Por su ligazón con este monográfico, podemos citar «Justicia social, interseccionalidad y profundización democrática» (Martínez-Palacios y Martínez-García), en el que tuvimos la oportunidad de iniciar esta reflexión colectiva sobre los desafíos y alternativas que plantea la relación entre estos términos.

Como no podía ser de otra manera, la publicación que tenemos entre manos ahonda en muchos de los aprendizajes que se generaron en ese espacio, aprovechando las sinergias que surgieron en esa publicación y en muchas otras iniciativas ligadas a una praxis investigadora transformativa. Pues la comprensión de la investigación y la educación como proceso, como relación en movimiento, es fundamental para seguir trabajando (no siempre con éxito y casi nunca desde las condiciones materiales y simbólicas deseables) en una práctica educativa e investigadora crítica y encaminada a la emancipación, como nos propone Patricia Hill Collins (Martínez-Palacios y Collins), cuyo marco inspira este monográfico.

En este sentido, la académica Feminista Negra nos orienta acerca de posibles vías para poner en diálogo interseccionalidad y democracia participativa desde un prisma de justicia social (Collins, The difference). De su mano podemos reflexionar sobre el hecho de que tanto los planteamientos de la democracia participativa como los de la interseccionalidad comparten una historia común, preocupaciones para la acción y, actualmente, enfrentan situaciones de peligro similares. Es decir, ambas propuestas se dirigen a la transformación social, cuestionando el modelo vigente de forma integral y radical, y promoviendo la desactivación de las lógicas de dominación en cada contexto. Pero, como se ha dicho, también comparten peligros. Amenazas que se pueden resumir en una banalización de su uso, en una institucionalización que encorseta y universaliza sus prácticas y, en general, en una pérdida de radicalidad en sus premisas e intenciones (Baiocchi y Ganuza; Collins y Bilge; Martínez-Palacios). Pues, como recuerda Collins (The difference 37):

Treating participatory democracy as a set of decontextualized principles that can be exported either into the academy or applied to preexisting state agendas misreads the significance of how and why freedom, justice, democracy and similar ethical ideas persist.

Además, la praxis interseccional le recuerda a las experiencias participativas que no están exentas de reproducir dominación a pesar de lo que dice 
su exposición declarativa. La participación ciudadana como herramienta de profundización democrática vive una extensión sin parangón, acogiendo multitud de formas, intereses y prácticas, que parece obligar a detenerse y analizar todos esos productos que se están generando (Bherer, Dufour y Montambeault). Hoy hablamos de giro participativo global para caracterizar la institucionalización de este interés en los procesos de toma de decisión participados. Ante este estallido, han sido las críticas feministas las que han alertado de los privilegios que perviven en estas estructuras, poniendo en evidencia que favorecen los discursos y las demandas de quienes han ocupado, tradicionalmente, el espacio público.

Sin embargo, se va evidenciando que fijarse únicamente en el género como eje que, por sí solo, explica la dominación, la invisibiliza y oculta, dificultando también el desarrollo de mecanismos que favorezcan su superación. Pues, como advierte Collins (Fighting 205), esta lectura exclusiva en términos de raza o género invisibiliza las relaciones injustas de poder que crean grupos sociales, ya que parte de un pensamiento binario (los hombres mandan sobre las mujeres, los blancos sobre los negros...). Bajo este marco, los grupos se definen como sujetos en oposición a otros que son objetivados en favor de quienes están en posición de privilegio (Collins, Black feminist 71), simplificando las formas en las que se produce la dominación. En palabras de bell hooks (37):

Si bien resulta evidente que muchas mujeres sufren la tiranía sexista, hay pocos indicios de que este hecho forje un «vínculo común entre todas las mujeres». Hay muchas pruebas que demuestran que las identidades de raza y clase crean diferencias en la calidad, en el estilo de vida y en el estatus social que están por encima de las experiencias comunes que las mujeres comparten; y se trata de diferencias que rara vez se transcienden.

De esta manera, la apuesta por la interseccionalidad que proponen estas pensadoras se presenta como una herramienta para enfocar de otras formas los problemas sociales y las respuestas a los mismos. Una necesidad de la que no se escapa la práctica participativa que, como alternativa crítica, se enfrenta también a las limitaciones que acarrea una interpretación monista o unitaria de los fenómenos sociales. Urge, por tanto, aprender de los paradigmas propuestos por las epistemologías críticas que desvelan la complejidad con la que se cruzan diferentes estructuras opresivas como la raza, la clase social,

Feminismo/s 33, junio 2019, pp. 13-20 
la edad, la diversidad funcional o la sexualidad, entre otras. Esta urgencia pretende ser tratada en este monográfico, en el que buscamos incorporar el marco de interpretación de Patricia Hill Collins (Black Feminist) desde la matriz de dominación, los ámbitos de poder y la idea de comunidad.

Así, inspirándose en este marco, los textos recogidos en este monográfico responden, desde disciplinas y planteamientos diversos, a la pregunta que subyace a la publicación que nos ocupa: ¿cómo podemos establecer los diálogos (qué pautas, qué ideas, que condicionantes, qué experiencias hay que tener en cuenta) entre democracia participativa e interseccionalidad para orientar la profundización democrática a la justicia social?

En primer lugar, el artículo que inaugura este trabajo colectivo, firmado por María Vanesa Ripio Rodríguez, abre la puerta a la reflexión sobre las potencialidades de la herramienta interseccional para cuestionar las concepciones pretendidamente universales de la democracia participativa. Desde la provocación filosófica, «Otro juego de herramientas: matriz de dominación y resistencia simbólica» hace conversar, principalmente, las propuestas de Patricia Hill Collins y Pierre Bourdieu aceptando el reto de la experimentación que le inspira Virgina Woolf. Desde estos posicionamientos, el artículo explora las posibilidades de la matriz de dominación para comprender, no solo las acciones y prácticas del mundo social, sino también de quienes se disponen a su conocimiento y análisis (la teórica o teórico social) y la propia definición de las reglas del juego (de la participación). Un ejercicio que contribuye a desvelar cómo se produce y reproduce la dominación en las distintas escalas pero, especialmente, a visibilizar y potenciar las posibilidades de resistencia entre los grupos oprimidos.

Justamente, la articulación de las identidades en el movimiento feminista es el objetivo que orienta el trabajo de Itziar Gandarias Goikoetxea, Marisela Montenegro Martínez y Joan Pujol Tarrés. Este texto asume el reto de plantear estrategias desde las que «descentrar» la perspectiva identitaria del concepto de interseccionalidad que ha desembocado en una suma despolitizada de discriminaciones que producen dos dinámicas: (1) el señalamiento unidireccional de la vulnerabilidad y (2) la fragmentación de las luchas sociales. A este respecto, en «Interseccionalidad, identidad y articulación: hacia una política de la agregación» se rechaza la premisa de que el reconocimiento de identidades es indisoluble de la división colectiva. Por el contrario, se apuesta 
por repolitizar la herramienta interseccional para tejer alianzas desde la diversidad, inspirándose en las propuestas de Jasbir Puar (ensamblajes), Gloria Anzaldúa (mundo zurdo) o Alejandra Castillo (la política de lo múltiple). Todas ellas entendidas como alternativas estratégicas, que ponen el punto de arranque en la enunciación de las diferencias, a la necesidad de articulación de los movimientos sociales tan presente en los tiempos actuales.

Por su parte, la identidad sigue siendo un elemento central en el tercer artículo del monográfico, en este caso, dirigiéndose a identidades mancilladas, que son consideradas como dañadas. Así, en el artículo «El estigma al servicio del poder. Dominación y resistencias desde la interseccionalidad» se plantea una comprensión compleja y estructural del estigma. Superando las interpretaciones individualistas y victimizantes de quienes reciben esta marca, Idoia del Hoyo analiza la estigmatización como proceso, atendiendo a las dinámicas macro y micro que interaccionan a partir del marco planteado por Collins. Desde la interseccionalidad, ofrece una imagen dinámica del estigma como fenómeno dependiente de los sistemas de dominación que intersectan en un contexto determinado para desentrañar sus causas particulares. Una mirada que posibilita hilar más fino en nuestras prácticas hacia una profundización democrática inclusiva, que problematiza las exclusiones de ciertos colectivos que se ven apartados de cualquier toma de decisiones desde esa condición de estigmatizados.

La perpetuación de los estigmas encuentra un aliado clave en los medios de comunicación, como parte del entramado en el que se negocia la ideología, la cultura y la conciencia (Collins, Black Feminist 283-284). El análisis del campo mediático se incorpora de la mano de Delicia Aguado-Peláez que examina el papel de la ficción televisiva como espejo y reflejo de la propia sociedad y de sus lógicas de dominación. La autora parte de que, en los últimos años, este espacio se ha ido abriendo a opciones contrahegemónicas, con producciones que ofrecen una representación de personajes más diversa, menos estereotipada y que pone en entredicho el imperio de ese sujeto normativo que invade las representaciones audiovisuales. Y, con ello, introduce nuevas temáticas y miradas que contribuyen a debilitar la violencia contra las mujeres (también la simbólica). Desde este punto de partida, «Violaciones en serie: dominaciones y resistencias. Las agresiones sexuales de ficción en la era del \#metoo», estudia cómo estas transformaciones (a ambos lados de

Feminismo/s 33, junio 2019, pp. 13-20 
la cámara) pueden influir en la representación de las violaciones, alejándose de patrones de monstruosidad individuales, problematizando lo estructural y poniendo a las mujeres (algunas mujeres) en el centro de la historia.

El quinto de los artículos que contiene este monográfico traslada la experimentación metodológica de la interseccionalidad a los movimientos sociales. A partir de sus investigaciones sobre la división del trabajo militante, Xavier Dunezat cuestiona las prácticas y las relaciones de poder que se producen en las luchas de sin-papeles en Francia, movimiento del que forma parte. Bajo el título «Las luchas de sin-papeles: entre comunidad prescrita, dispersión real y profundización democrática», el autor visibiliza la existencia de formas legitimadas de militancia que excluyen las acciones de los propios sujetos protagonistas de la opresión. De esta manera, problematiza la reproducción de lógicas de dominación en los espacios alternativos. Asimismo, también cuestiona la normatividad de los liderazgos, que desvaloriza las formas de expresión y acción que no concuerdan con ese militante ideal (por tanto, imbuido de privilegio). Y, con ello, propone estrategias que posibiliten alianzas en términos de horizontalidad y reconocimiento entre quienes participan de las luchas encaminadas a la justicia social.

Trasladando el foco de Francia al País Vasco, Igor Ahedo y Miriam Ureta se suman a la comprensión compleja de las vulnerabilidades (y los privilegios) para poder desarrollar prácticas participativas realmente inclusivas. En «Desvelando opresiones y resistencias de las mujeres en Zumarraga: un análisis interseccional para profundizar la democracia participativa en términos de justicia social», incorporan la advertencia de Collins (The difference 37) de que los proyectos de interseccionalidad y democracia no pueden ser desligados de sus contextos históricos y sociales específicos. Así, quienes firman este artículo parten de una situación de desmantelamiento industrial que impacta en las formas de vida de este municipio del interior de Gipuzkoa. $\mathrm{Y}$, especialmente, en las de las mujeres cuyas problemáticas han sido invisibilizadas y desconsideradas en un modelo que llora la desaparición del clásico ganador de pan. En este sentido, este texto contribuye a la visibilización de las necesidades diversas de las habitantes de Zumarraga, apostando por el reconocimiento de sus presencias y voces, como primer paso para el cambio político y social de la localidad en su apuesta por la participación ciudadana. 
Finalmente, el último de los trabajos que presenta el monográfico examina las transformaciones que se han producido en las sociedades pesqueras a raíz de la profesionalización de los trabajos feminizados. En concreto, del marisqueo a pie. En «Cuando las mujeres acceden al poder. Discriminaciones y resistencias en las comunidades marineras» se presentan las experiencias vividas por las mujeres que han accedido a puestos de responsabilidad en las organizaciones pesqueras. En el texto, Patricia Martínez-García expone las distintas formas de opresión que viven las mariscadoras cuando se integran en espacios de poder diseñados por y para los hombres. Desde los modos de expresión y las actitudes hasta la gestión de las cofradías de pescadores o las propias exigencias temporales del cargo que dificultan la conciliación. No obstante, la creciente presencia femenina ha venido a cuestionar (ya para quedarse) los mecanismos que han operado en estos lugares, convirtiéndose en un sujeto estratégico para la revitalización del sector pesquero y de sus comunidades.

En definitiva, todos y cada uno de estos artículos son una exploración de las potencialidades del diálogo entre la interseccionalidad y la democracia participativa, entendidas desde su práctica radical. Desde diferentes miradas, disciplinas y planteamientos, quienes firman los textos de este monográfico cuestionan estructuras, conceptos y acciones, y proporcionan alternativas para repensar nuestros entornos en una clave de justicia social. Una experiencia colectiva desde la que también se reivindica un posicionamiento crítico de la investigación como paso previo a la emancipación y transformación en nuestras sociedades.

\section{REFERENCIAS BIBLIOGRÁFICAS}

Baiocchi, Gianpaolo y Ernesto Ganuza. Popular democracy. The paradox of participation. Stanford: Stanford University Press, 2016.

bell hooks. «Mujeres negras. Dar forma a la teoría feminista. Otras inapropiables. Feminismos desde las fronteras. Eds. bell hooks, Avtar Brah, Chela Sandoval, Gloria Anzaldúa, Aurora Levins Morales, Kum-Kum Bhavani, Margaret Coulson, M. Jaqui Alexander y Chandra Talpade Mohanty. Madrid: Traficantes de Sueños, 2004. 33-50. 
Bherer, Laurence, Pascale Dufour y Françoise Montambeault. «The participatory democracy turn: an introduction». Journal of civil society 12.3 (2016): 225-230.

Collins, Patricia Hill. Black feminist thought. Knowledge, consciousness, and the politics of empowerment. Segunda edición. New York: Routledge, 2000.

-. Fighting words. Black Women and the search for justice. Minneapolis: University of Minnesota, 1998.

—. «The difference that power makes». Investigaciones feministas 8.1 (2017): $19-40$.

Martínez-Palacios, Jone. «Problemas de la institucionalización y la profesionalización de la participación en contextos de profundización democrática». Revista Internacional de Sociología 76.1 (2018). 6 junio 2019.

Martínez-Palacios, Jone y Patricia Hill Collins. «Educación crítica y emancipadora desde la epistemología del feminismo negro». Hariak. Recreando la educación emancipadora 4 (2018): 12-19.

Martínez-Palacios, Jone y Patricia Martínez-García. «Justicia social, interseccionalidad y profundización democrática». Investigaciones Feministas, 8.1 (2017). 